Supporting Information for

\title{
Selective Determination of Isothermally Amplified Zika Virus RNA Using a Universal DNA-Hairpin Probe in Less than 1 hour
}

\author{
Charles A. Lynch IIIț, , Marcos V. Foguelța, Adam J. Reed ${ }^{a}$, Angelica M. Balcarcela, Percy \\ Calvo-Marzal $^{\text {a }}$, Yulia V. Gerasimova ${ }^{\text {, }}$ Karin Y. Chumbimuni-Torres ${ }^{\text {a }}$ \\ ${ }^{a}$ Department of Chemistry, University of Central Florida, 4000 Central Florida Blvd., Orlando, FL 32816, United \\ States \\ ${ }^{b}$ Rose Hulman Institute of Technology, 5500 Wabash Ave., Terre Haute, IN 47803, United States
}




\section{Sequences of the RNA/DNA}

Table S1. Oligonucleotides sequences* used in 4WJ-based biosensor and NASBA.

\begin{tabular}{|c|c|}
\hline Oligonucleotide & Sequence (5'-3') \\
\hline UDH probe & /ThiolMC6-D/TTTTTTTTTTCGCGTTAACATACAATAGATCGCG \\
\hline m-strand & СTCCCCTTCT/iSp9/TATGTTAAC TTTTTTTTTT-MеB \\
\hline f-strand & GATCTATTG/iSp9/ACTGATCTCCACATGATGTTT \\
\hline ZIKV T-138 & $\begin{array}{l}\text { GCAGTCAAGCAAGCCTGGGAAGATGGTATCTGCGGGATCTCCTCTGTTTCAAGAATGGA } \\
\text { AAACATCATGTGGAGATCAGTAGAAGGGGAGCTCAACGCAATCCTGGAAGAGAATGGA } \\
\text { GTTCAACTGACGGTAGTTGTG }\end{array}$ \\
\hline DENV T-114 & $\begin{array}{l}\text { CAGATCTCTGATGAACAACCAACGGAAAAAGACGGGTAGACCGTCTTTCAATATGCTGA } \\
\text { AACGCGCGAGAAACCGCGTGTCAACTGTTTCACAGTTGGCGAAGAGATTCTCAAA }\end{array}$ \\
\hline Forward primer & АATTCTAATACGACTCACTATAGGGAGAAGGAGATTGGCAGCAGCWGTYAAGCA \\
\hline Reverse primer & CACAACGACCGTCAGTTGAA \\
\hline $\begin{array}{l}\text { NASBA } \\
\text { amplicon }\end{array}$ & $\begin{array}{l}\text { GGGAGAAGGAGAUUGGCAGCAGCAGUCAAGCAAGCCUGGGAAGAUGGUAUCUGCGGG } \\
\text { AUCUCCUCUGUUUCAAGAAUGGAAAACAUCAUGUGGAGAUCAGUAGAAGGGGAGCUC } \\
\text { AACGCAAUCCUGGAAGAGAAUGGAGUUCAACUGACGGUCGUUGUG }\end{array}$ \\
\hline
\end{tabular}

*ThiolMC6-D: 5' Thiol Modifier C6 S-S; MeB: Methylene Blue; iSp9: Triethylene glycol spacer. Fragments of the probes/targets complementary to each other are color coded.

\section{Analysis of NASBA amplicons in agarose gel}

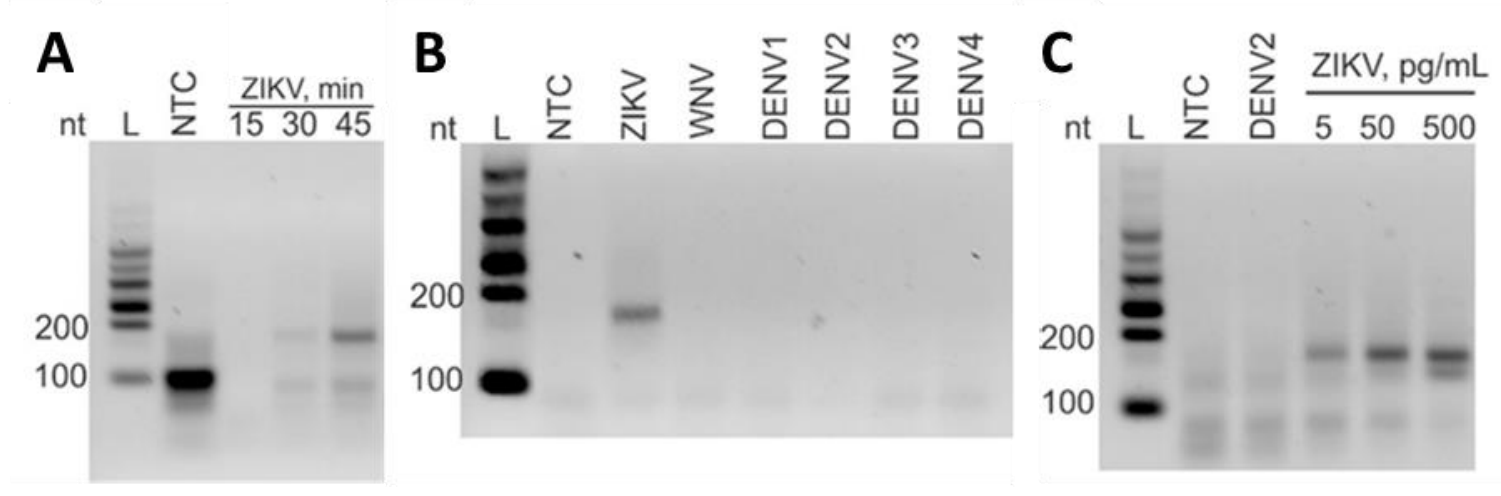

Figure S1. Analysis of NASBA amplicons in $2 \%$ agarose gel. (A) Products of NASBA of 50 fg/ $\mu \mathrm{L}$ ZIKV RNA

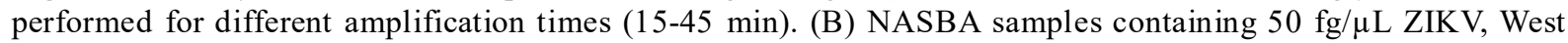
Nile virus (WNV), or each of the four serotypes of dengue virus (DENV1-4) were incubated for $45 \mathrm{~min}$. (C) Concentration of ZIKV RNA in NASBA reactions were 5, 50 and $500 \mathrm{fg} / \mu \mathrm{L}$ amplified for $45 \mathrm{~min}$. L: ssRNA ladder (the bands corresponding to 100- and 200-nt RNA fragments are labeled); NTC: contained no viral RNA added.

It should be noted that in some cases a band corresponding to about 100 -nt RNA product was observed in gel for NTC NASB A sample (Figure S1-A). Despite this, no signal higher than that of the blank was detected for such NTC sample (Figure 3) using the 4WJ-based biosensor, which further proves high selectivity of the target recognition by the biosensor. 


\section{Biosensor response using synthetic DNA}

The biosensor optimization was performed using ZIKV T-138 target, which mimics a targeted fragment of the ZIKV genome and contains the sequence complementary to the target-binding fragments of $\mathrm{m}$-strand and f-strand (Table S1). First, the time required for hybridization of the target and adaptor strands to the electrode immobilized universal DNA-Hairpin (UDH) probe was optimized. The adaptor strands and target were allowed to interact with the UDH probe for $1,2.5,5,10,20,30,60$ or 90 min before the current density peak ( $\mathrm{j}_{\mathrm{p}}$ ) was measured (Figure $\mathrm{S} 2)$. The average current density peak of $14 \pm 2 \mu \mathrm{A} / \mathrm{cm}^{2}$ triggered by $50 \mathrm{nM}$ ZIKV T-138 was obtained for a 90 min hybridization time. Moreover, a measurable signal $\left(0.8 \pm 0.2 \mu \mathrm{A} / \mathrm{cm}^{2} \mathrm{vs}\right.$ $0.36 \pm 0.03 \mu \mathrm{A} / \mathrm{cm}^{2}$ for the blank sample measured in the absence of the target) can be observed almost immediately (within $1 \mathrm{~min}$ ) after addition of the target and adaptor strands. A 10-fold increase of the signal $\left(3.7 \pm 0.1 \mu \mathrm{A} / \mathrm{cm}^{2}\right)$ over blank was observed for 10 min hybridization time. Such difference is high enough to reliably distinguish the signal triggered in the target's presence from the blank. Thus, in order to reduce the detection time, 10 minutes was used for further experiments.

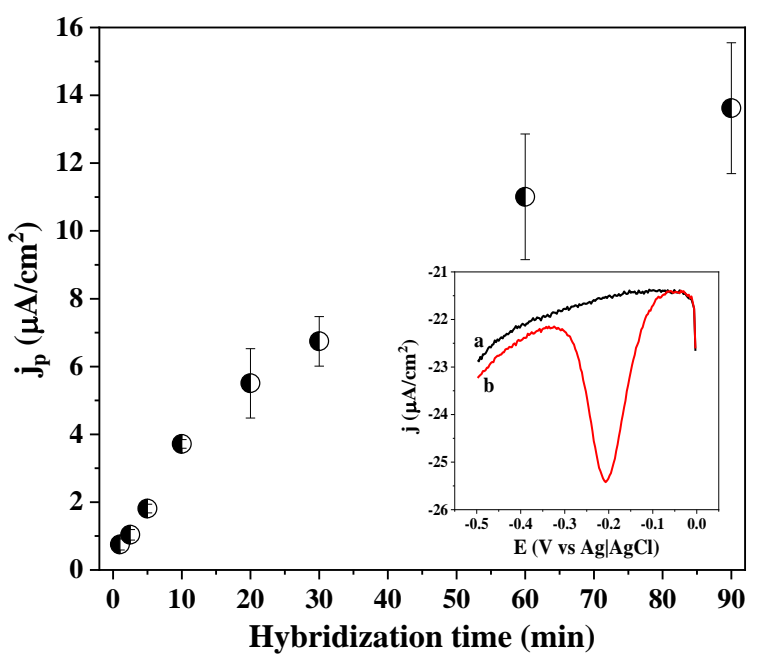

Figure S2. Biosensor response at a varied hybridization time $(1,2.5,5,10,20,30,60$ and $90 \mathrm{~min}) \mathrm{using} 50 \mathrm{nM}$ ZIKV T-138. Inset: SWV response of a GDE/UDH with MCH backfill layer before (a) and after (b) addition of 50 nM ZIKV T-138, $0.5 \mu \mathrm{M}$ f-strand and $0.25 \mu \mathrm{M}$ m-strand, and incubation for $10 \mathrm{~min}$.

After choosing an optimal hybridization time, control experiments were conducted to verify the response of the biosensor in the absence of the target and/or other components of the $4 \mathrm{WJ}$ ensemble. The results can be observed in Figure S3 along with the current density peak for the GDE-immobilized 4WJ structure formed in the presence of 5 or $50 \mathrm{nM} \mathrm{ZIKV} \mathrm{T-138.} \mathrm{These}$ results demonstrate that the biosensor needs all four $4 \mathrm{WJ}$ components to provide a discernable current density peak. All $4 \mathrm{WJ}$ components without the target sequence (3) resulted in a signal of $0.36 \pm 0.03 \mu \mathrm{A} / \mathrm{cm}^{2}$, which establishes the blank response of the biosensor. The current density peak obtained from the control experiments were all within the error of the average signal for the blank sample. Without the target and f-strand (4), the current density peak was determined to be $0.31 \pm 0.03 \mu \mathrm{A} / \mathrm{cm}^{2}$, which was within the error of the blank biosensor response. The $5 \mathrm{nM}$ concentration of ZIKV T-138 (5) was determined to be $0.55 \pm 0.09 \mu \mathrm{A} / \mathrm{cm}^{2}$, which was outside the range of the blank responses. While higher concentrations, as $50 \mathrm{nM}$ 
ZIKV T-138 (6), demonstrate a significant discrimination compared to blank responses $(3.7 \pm$ $0.1 \mu \mathrm{A} / \mathrm{cm}^{2}$ against $\left.0.36 \pm 0.03 \mu \mathrm{A} / \mathrm{cm}^{2}\right)$.

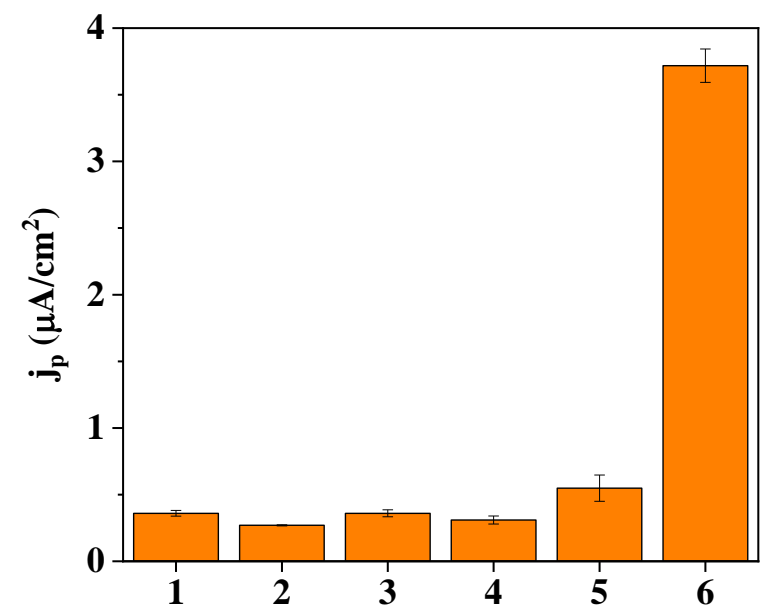

Figure S3. Biosensor response for (1) strands m, f and ZIKV T-138 target (absence of UDH); (2) UDH probe, strands $\mathrm{m}$ and target (absence of strand $\mathrm{f}$ ); (3) UDH probe, strands $\mathrm{m}$ and $\mathrm{f}$ (absence of target); (4) UDH probe and strand $\mathrm{m}$ (absence of strand $\mathrm{f}$ and target); and UDH probe, strands $\mathrm{m}$ and $\mathrm{f}$ and target at $5 \mathrm{nM}$ (5) and 50 $\mathrm{nM}$ (6). 10 min of hybridization time was used for all samples.

To analyze hybridization between the UDH probe, strands $\mathrm{m}$ and $\mathrm{f}$, and the target, we performed native polyacrylamide gel electrophoresis of the individual oligonucleotides and their mixtures prepared in the hybridization buffer to imitate the conditions of electrochemical analysis (Figure S4). As can be seen by comparing the bands in lane 4 with those in lanes 5-7, the UDH probe, strand $\mathrm{f}$, and strand $\mathrm{m}$ show no interaction in the absence of the target. This is in agreement with the negligible signal of the electrochemical biosensor in the absence of the target (Figure S3, bar 3). A low-mobility band at $\sim 150$ bp (indicated with an arrow in Figure S4) can be observed in lane 2 containing a sample with all four components of $4 \mathrm{WJ}$ present (the sample produces high signal in the case of electrochemical biosensor response, Figure S3, bar 6). This band is missing if the UDH probe is omitted (Figure S4, lane 3). Strands $\mathrm{f}$ and $\mathrm{m}$ interrogate the target to form partial complexes (Figure S4, lane 3, two more intense bands right below the band indicated with an arrow), but the UDH probe is required for the complete assembly. The difference in mobility between the arrow-labeled band (lane 2) and the lowest mobility band in lane 3 is about $25 \mathrm{bp}$, which corresponds to the difference in theoretical sizes between complete $4 \mathrm{WJ}$ complex and a tricomponent $\mathrm{m} / \mathrm{f} / \operatorname{target}$ complex (18 bp plus $16 \mathrm{nt}$ ). Therefore, we speculate that the lowest mobility band in lane 2 corresponds to the $4 \mathrm{WJ}$ structure formed between the UDH probe, strands $\mathrm{m}$ and $\mathrm{f}$, and the target. As expected, the complex formation in solution is not very efficient, since the strands were designed to work on the electrode surface. 


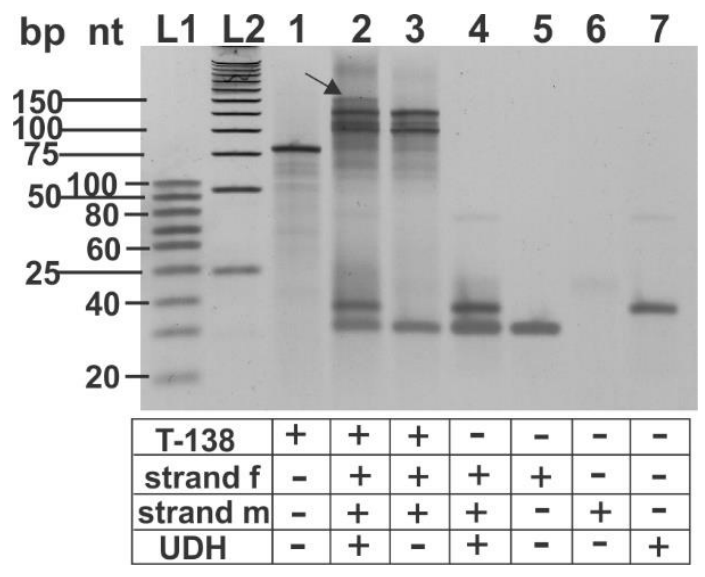

Figure S4. Electrophoretic analysis of the $4 \mathrm{WJ}$ complex formation in $8 \%$ polyacrylamide gel supplemented with $12.5 \mathrm{mM} \mathrm{MgCl}_{2}$ under native conditions. The samples containing the indicated components were annealed in the hybridization buffer at $6 \mu \mathrm{M}$ concentration of UDH, strands $\mathrm{m}$ and $\mathrm{f}$, and $3 \mu \mathrm{M} \mathrm{T}-138$. The samples were then diluted 12-fold in the solution containing 1x Blue/Orange Loading Dye (Promega, Madison, WI), with the concentration of $\mathrm{MgCl}_{2}$ adjusted to $12.5 \mathrm{mM}$, and $7 \mu \mathrm{L}$ of each sample was loaded on the gel. The gel was run at $15 \mathrm{~V} / \mathrm{cm}$ at $10^{\circ} \mathrm{C}$. The bands were visualized by staining with GelRed (Biotium, Fremont, CA). Lanes L1 and L2 contained 20/100 ssDNA ladder (IDT, Coralville, IA) and 25 bp step DNA ladder (Promega, Madison, WI), respectively. The sizes of the bands in both ladders are indicated to the left. The position of the $4 \mathrm{WJ}$ complex containing T-138, strands $\mathrm{m}$ and $\mathrm{f}$, and USL is shown with an arrow.

The calibration curves obtained for the biosensor response to varied concentration of ZIKV T-138 from 1 to $75 \mathrm{nM}$ is shown in Figure S5. The sensitivity of the biosensor was 0.068 $\mu \mathrm{A} / \mathrm{cm}^{2} \mathrm{nM}$. The limit of detection (LOD) was calculated as LOD $=3^{*} \sigma / \mathrm{S}$, where $\sigma$ is the standard deviation of the blank and $\mathrm{S}$ is defined as the slope of the calibration curve. The LOD of the biosensor (for T-138 mimicking the NASBA product) was $1.24 \mathrm{nM}$.

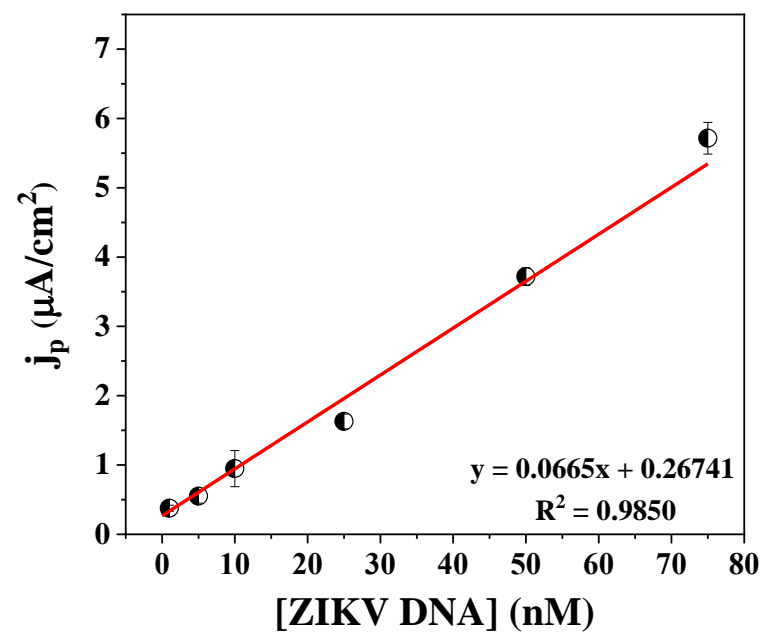

Figure S5. Biosensor response to varied concentrations of ZIKV T-138 at 1, 5, 10, 25, 50 and 75 nM and with 10 min of hybridization time.

The selectivity of the biosensor was first evaluated using DENV T-114 as a target. In Figure S6, the SWV responses are shown before the hybridization step (curve a), after hybridization of the UDH probe with the adaptor strands and either $50 \mathrm{nM}$ DENV T-114 (curve b) or $50 \mathrm{nM}$ ZIKV T-138 (curve c). The current density peak triggered by the ZIKV DNA target is substantially larger than by the DENV DNA target of the same concentration. The average 
current density peak obtained with $50 \mathrm{nM}$ DENV DNA was $0.16 \pm 0.04 \mu \mathrm{A} / \mathrm{cm}^{2}$, while a signal of $3.2 \pm 0.4 \mu \mathrm{A} / \mathrm{cm}^{2}$ was observed in the presence of $50 \mathrm{nM}$ ZIKV DNA. Thus, the biosensor displays high selectivity with the ability to differentiate between Zika and Dengue viruses.

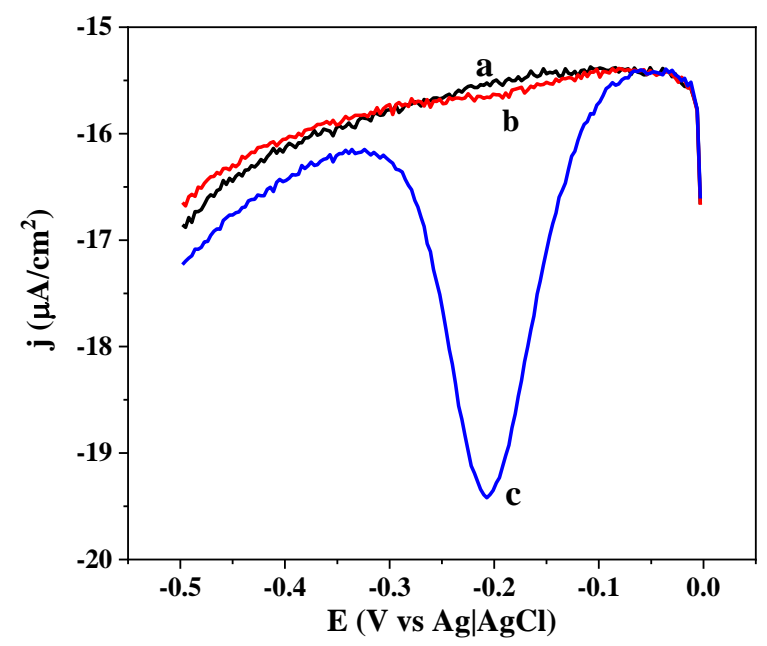

Figure S6. SWV response of a GDE/UDH with MCH backfill layer (a), after incubation of the electrode with the adaptor strands and either $50 \mathrm{nM}$ DENV T-114 (b) or $50 \mathrm{nM}$ ZIKV T-138 (c), both with the incubation time of 10 $\min$. 